\title{
DSpace@MIT
}

\author{
MIT Open Access Articles
}

\section{Preparing topological projected entangled pair states on a quantum computer}

The MIT Faculty has made this article openly available. Please share how this access benefits you. Your story matters.

Citation: Schwarz, Martin, Kristan Temme, Frank Verstraete, David Perez-Garcia, and Toby S. Cubitt. "Preparing topological projected entangled pair states on a quantum computer." Physical Review A 88, no. 3 (September 2013). (c) 2013 American Physical Society

As Published: http://dx.doi.org/10.1103/PhysRevA.88.032321

Publisher: American Physical Society

Persistent URL: http://hdl.handle.net/1721.1/84649

Version: Final published version: final published article, as it appeared in a journal, conference proceedings, or other formally published context

Terms of Use: Article is made available in accordance with the publisher's policy and may be subject to US copyright law. Please refer to the publisher's site for terms of use. 


\title{
乌ु \\ Preparing topological projected entangled pair states on a quantum computer
}

\author{
Martin Schwarz, ${ }^{1}$ Kristan Temme, ${ }^{2}$ Frank Verstraete, ${ }^{1}$ David Perez-Garcia, ${ }^{3}$ and Toby S. Cubitt $3{ }^{3,4}$ \\ ${ }^{1}$ Vienna Center for Quantum Science and Technology, Faculty of Physics, University of Vienna, Vienna, Austria \\ ${ }^{2}$ Center for Theoretical Physics, Massachusetts Institute of Technology, 77 Massachusetts Avenue, Cambridge, \\ Massachusetts 02139-4307, USA \\ ${ }^{3}$ Departamento de Análisis Matemático, Universidad Complutense de Madrid, Plaza de Ciencias 3, Ciudad Universitaria, \\ 28040 Madrid, Spain \\ ${ }^{4}$ DAMTP, University of Cambridge, Centre for Mathematical Sciences, Wilberforce Road, Cambridge CB3 OWA, United Kingdom
}

(Received 6 February 2013; published 23 September 2013)

\begin{abstract}
Simulating exotic phases of matter that are not amenable to classical techniques is one of the most important potential applications of quantum information processing. We present an efficient algorithm for preparing a large class of topological quantum states, the $G$-injective projected entangled pair states (PEPS), on a quantum computer. Important examples include the resonant valence bond states, conjectured to be topological spin liquids. The runtime of the algorithm scales polynomially with the condition number of the PEPS projectors and inverse polynomially in the spectral gap of the PEPS parent Hamiltonian.
\end{abstract}

DOI: 10.1103/PhysRevA.88.032321

PACS number(s): 03.67.Ac, 03.65.Vf, 03.67.Lx

\section{INTRODUCTION}

Creating and studying exotic phases of matter is one of the most challenging goals in contemporary physics. The increasingly sophisticated simulation abilities of systems such as cold atoms in optical lattices, trapped ions, or superconducting qubits make this accessible by means of Feynmann's original idea of using highly controllable quantum systems to simulate other quantum systems. Among those exotic phases, non-Abelian topologically ordered states and topological spin liquids, such as resonating valence bond (RVB) states in frustrated lattices, are probably the holy grails of this area of quantum state engineering. Progress on the creation of such exotic phases in various experimental systems has accelerated rapidly in recent years, including cold atoms [1], ion traps [2], photonic devices [3], and superconducting devices [4].

Recently [5], a very general way of constructing quantum states on a quantum computer was proposed. The wide applicability of the method lies in the fact that there is a variational class of quantum states, called projective entangled pair states (PEPS) [6], which has a simple local description but is nonetheless complex enough to approximate the low-energy sector of local Hamiltonians. (A review of the analytical and numerical evidence for this can be found in [7] and the references therein.) However, a crucial technical assumption in the main result of [5], called "injectivity," excludes any possibility of constructing quantum states with topological order.

The main aim of this article is to significantly extend these results to include exotic topological quantum phases, by proving the following.

Main result. Any $G$-injective PEPS can be prepared on a quantum computer in polynomial time, when the spectral gap of the associated parent Hamiltonian scales at most inverse polynomially in the system size.

" $G$-injectivity", introduced only recently in [7] (and explained more fully below), is a substantially weaker requirement than injectivity, which explicitly allows for topological order. A compelling example of the significance of this result is the very recently proven fact that the RVB state in the kagome lattice (conjectured to be a topological spin liquid) is a $\mathbb{Z}_{2}$-injective PEPS, with numerical evidence that the gap assumption is also verified [8]. Our result therefore gives one way in which the RVB state (and other topological states) can be prepared efficiently on a general quantum simulator. A large class of topological states is captured by quantum-double models [9]. These are equivalent to $G$-isometric PEPS [10] and easy to prepare [11], which is related to the fact that the terms of the respective parent Hamiltonians always commute. $G$-injective PEPS generalize $G$-isometric PEPS and capture an even larger class of topological quantum states which are ground states of parent Hamiltonians with noncommuting terms for which no efficient preparation procedure has been known before. Engineering exotic quantum states by quantum simulation complements research aimed at finding materials that directly exhibit topological behavior, and is already beginning to bear fruit experimentally [1-4].

In the following section, we summarize basic notions of PEPS required in this work, and introduce the class of $G$-injective PEPS which includes many of the important topological quantum states. We then briefly review the algorithm of Ref. [5] for preparing injective (nontopological) PEPS before proceeding to show how this algorithm can be extended to the much larger class of $G$-injective PEPS, thereby allowing efficient preparation of many exotic topological quantum states. Finally, we close with some concluding remarks and open questions.

Projected entangled pair states. For simplicity, we will focus on PEPS defined on a square lattice, but the results can be generalized to other lattices. An (unnormalized) PEPS can be described as follows. Place maximally entangled states of dimension $D$ along all edges of the lattice. To each vertex $v$, apply a linear map $A^{v}:\left(\mathbb{C}^{D}\right)^{\otimes 4} \rightarrow \mathbb{C}^{d}$ to the four $D$-dimensional systems labeled $l, t, r, b$ (for left, top, right, and bottom, respectively), where $A^{v}=$ $\sum_{i: l, t, r, b} A_{i: l t r b}^{v}|i\rangle\langle l t r b|$. The resulting vector is the unnormalized PEPS. Since local unitaries do not change the complexity of preparing a state, for the purposes of this work we can assume without loss of generality that $A$ is positive semidefinite, by taking its polar decomposition. When $A$ is left invertible, we call the PEPS injective [7]. 
A particularly interesting class of PEPS is the class of $G$-isometric PEPS, defined for any finite group $G$ as follows. Take a semiregular representation of $G$ [7], that is, a representation $U_{g}=\oplus_{\alpha} V_{g}^{\alpha} \otimes \mathbb{1}_{r_{\alpha}}$ having at least one copy of each irrep $\alpha$. Note that the regular representation is exactly the one for which $r_{\alpha}$ is the dimension $d_{\alpha}$ of the irrep $V_{g}^{\alpha}$ for all $\alpha$. We can define the reweighting map

$$
\Delta=\oplus_{\alpha}\left(\frac{d_{\alpha}}{r_{\alpha}}\right)^{\frac{1}{4}} \mathbb{1}_{d_{\alpha}} \otimes \mathbb{1}_{r_{\alpha}},
$$

which is real, diagonal, commutes with $U_{g}$, and satisfies $\operatorname{Tr} \Delta^{4} U_{g}=|G| \delta_{g, e}$. (For the regular representation, $\Delta=\mathbb{1}$.) The PEPS is then defined by taking, for all $\nu, A^{\nu}=\frac{1}{|G|} \sum_{g \in G} \Delta \bar{U}_{g} \otimes \Delta \bar{U}_{g} \otimes \Delta U_{g} \otimes \Delta U_{g} . G$-isometric PEPS were originally defined in [7] only for the regular representation, and shown to be exactly the quantum-double models of Kitaev [9]. We use the argument described in [7] for the toric code and RVB states, generalized here to arbitrary $G$-isometric PEPS, to see that the $G$-isometric PEPS for any semiregular representation is equivalent to the one for the regular representation. Let us start with a semiregular representation $U_{g}$ of a group $G$, and let

$$
B=\frac{1}{|G|} \sum_{g} \Delta \bar{U}_{g} \otimes \Delta \bar{U}_{g} \otimes \Delta U_{g} \otimes \Delta U_{g} .
$$

We will show how $B$ can indeed be seen as the $G$-isometric PEPS corresponding to the regular representation, possibly composed with an isometry which embeds the initial Hilbert space into a sufficiently large one. The latter can be prepared efficiently on a quantum computer by other means [11], which will be discussed in more detail in Sec. II.

To show this, we decompose the tensor $B$ into two tensors of the form $A=(\sqrt{|G|})^{-1} \sum_{g} \Delta U_{g} \otimes \Delta U_{g} \otimes|g\rangle$ [where $U_{g}$ and $U_{g}$ are interchanged as needed, as shown in Fig. 1(a)]. By regrouping these new tensors, we obtain a new PEPS decomposition of the same state, where now the bond

(a)

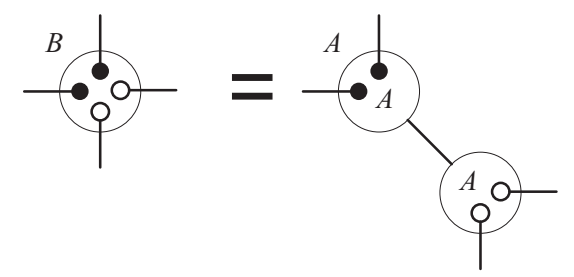

(b)

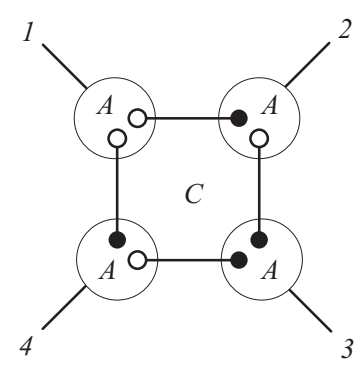

FIG. 1. (a) Illustrates the decomposition of the original tensor in the tensors $A$. We mark in white the bonds in which we have $U_{g}$ and in black those in which we have $\bar{U}_{g}$. (b) Illustrates the new way of grouping the tensors to get a $G$-isometric PEPS, called $C$. The bonds of this new tensor are numbered clockwise as in the figure. dimension is $|G|$ [Fig. 1(b)]. The resulting tensor $C$ [Fig. 1(c)], as a map from the virtual to the physical indices, is given by

$$
\begin{aligned}
C:\left|g_{1} g_{2} g_{3} g_{4}\right\rangle \mapsto & \frac{1}{|G|^{2}} \Delta^{2} U_{g_{1} g_{2}^{-1}} \otimes \Delta^{2} U_{g_{2} g_{3}^{-1}} \\
& \otimes \Delta^{2} U_{g_{4} g_{3}^{-1}} \otimes \Delta^{2} U_{g_{1} g_{4}^{-1}} .
\end{aligned}
$$

By calling $g=g_{1}^{-1} g_{1}^{\prime}$ and using Eq. (1) it is straightforward to see that

$$
\begin{aligned}
& \left\langle g_{1}^{\prime} g_{2}^{\prime} g_{3}^{\prime} g_{4}^{\prime}\left|C^{\dagger} C\right| g_{1} g_{2} g_{3} g_{4}\right\rangle \\
& =\frac{1}{|G|^{4}} \prod_{r=1}^{2} \operatorname{Tr}\left(\Delta^{4} U_{g_{r} g_{r+1}^{-1} g_{r+1}^{\prime} g_{r}^{\prime}}\right) \prod_{r=3}^{4} \operatorname{Tr}\left(\Delta^{4} U_{g_{r+1} g_{r}^{-1} g_{r}^{\prime} g_{r+1}^{\prime}-1}\right)
\end{aligned}
$$

equals 1 if and only if there exist $g$ such that $g_{i} g=g_{i}^{\prime}$ for all $i$. Otherwise, the expression is identically zero. Therefore, $C^{\dagger} C=(|G|)^{-1} \sum_{g} R_{g}^{\otimes 4}$ for the regular representation $R_{g}$, hence the new PEPS $C$ is the $G$-isometric PEPS corresponding to the regular representation.

If, on top of a $G$-isometric PEPS, we apply a further invertible (and w.l.o.g. positive-definite) linear map $A^{v}$ : $\mathbb{C}^{d} \rightarrow \mathbb{C}^{d}$, we obtain a " $G$-injective" PEPS [7]. (Here, $d$ is the dimension of the symmetric subspace associated with the group.) The parallel with plain injective PEPS is clear. Both are defined by invertible maps on top of a $G$-isometric PEPS. In the case of injective PEPS, the group is the trivial one and the representation is simply $\mathbb{1}_{d}$ (d copies of the left-regular representation of the trivial group).

$G$-isometric PEPS have very nice properties, coming from their topological character, which are inherited by the more general $G$-injective PEPS. For instance, for each $G$-isometric PEPS $|\psi\rangle$ there exists a local frustration-free Hamiltonian (called the PEPS "parent Hamiltonian" [7]), consisting of commuting projectors and having as ground space the subspace (over)spanned by $\{|\psi ; K\rangle: K=(g, h),[g, h]=0\}$. (Here, $|\psi ; K\rangle$ is the PEPS obtained by the same maps $A$, except that we first apply an additional $U_{g}^{\otimes V}$ to exactly one vertical strip $V$ and $U_{h}^{\otimes H}$ to exactly one horizontal strip $H$ in the initial collection of maximally entangled states [7].) This generalizes to $G$-injective PEPS, except that the local Hamiltonian terms are no longer necessarily commuting projectors.

We will denote by $\left|A^{1} \ldots A^{t}\right\rangle$ the $G$-injective PEPS defined by applying the map $A^{j}$ to vertex $j$ for $j=1, \ldots, t$ (and identity to the rest of the vertices) on top of the $G$-isometric PEPS, and define the states $\left|A^{1} \ldots A^{t} ; K\right\rangle$ analogously to above, which again (over)span the ground space of a frustration-free local parent Hamiltonian $H_{t}$.

\section{ALGORITHM}

\section{A. Preparing injective PEPS}

We first briefly review the algorithm of [5] for preparing injective PEPS on a quantum computer. Let $H_{t}$ be the parent Hamiltonian of the partially constructed state $\left|A^{1} \ldots A^{t}\right\rangle$. The algorithm starts at $t=0$ with maximally entangled states between all pairs of adjacent sites in the lattice, and proceeds by successively projecting onto the ground states of $H_{t}$ for $t=1 \ldots N$ until the final state $\left|A^{1} \ldots A^{N}\right\rangle$ is reached. 
Since the ground state $P_{t}$ of $H_{t}$ is a complex, many-body quantum state, it is not immediately clear (i) how to efficiently perform the projective measurement $\left\{P_{t}, P_{t}^{\perp}\right.$ \}onto the ground state. Furthermore, measurement in quantum mechanics is probabilistic, so even if this measurement can be performed, it is not at all clear (ii) how to guarantee the desired outcome $P_{t}$.

The answer to (i) is to run the coherent quantum phase estimation algorithm $[12,13]$ for the unitary generated by time evolution under $H_{t}$. (Time evolution under the local Hamiltonian $H_{t}$ can be simulated efficiently by standard Hamiltonian simulation techniques [14].) If $\sum_{k} \alpha_{k}\left|\psi_{k}\right\rangle$ is the initial state expanded in the eigenbasis of $H_{t}$, then the phase estimation entangles this register with an output register containing an estimate of the corresponding eigenvalue: $\sum_{k} \alpha_{k}\left|\psi_{k}\right\rangle\left|E_{k}\right\rangle$. Performing a partial measurement on the output register to determine if its value is less than $\Delta_{t}$ (the spectral gap of $H_{t}$ ) completes the implementation of the measurement $\left\{P_{t}, P_{t}^{\perp}\right\}$. (See [5] for full details.)

The solution to (ii) is more subtle, and makes use of Jordan's lemma of 1875 on the simultaneous block diagonalization of two projectors, which we first recall:

Lemma $l$ (Jordan [15]). Let $R$ and $Q$ be projectors with rank $s_{r}$ and $s_{q}$, respectively. Then, both projectors can be decomposed simultaneously in the form $R=\bigoplus_{k=1}^{s_{r}} R_{k}$ and $Q=\bigoplus_{k=1}^{s_{q}} Q_{k}$, where $R_{k}, Q_{k}$ denote rank-1 projectors acting on one- or two-dimensional subspaces. The eigenvectors $\left|r_{k}\right\rangle,\left|r_{k}^{\perp}\right\rangle$ and $\left|q_{k}\right\rangle,\left|q_{k}^{\perp}\right\rangle$ of the $2 \times 2$ projectors $R_{k}$ and $Q_{k}$ are related by $\left|r_{k}\right\rangle=$ $\sqrt{d_{k}}\left|q_{k}\right\rangle+\sqrt{1-d_{k}}\left|q_{k}^{\perp}\right\rangle,\left|r_{k}^{\perp}\right\rangle=-\sqrt{1-d_{k}}\left|q_{k}\right\rangle+\sqrt{d_{k}}\left|q_{k}^{\perp}\right\rangle$, $\left|q_{k}\right\rangle=\sqrt{d_{k}}\left|r_{k}\right\rangle-\sqrt{1-d_{k}}\left|r_{k}^{\perp}\right\rangle$, and $\left|q_{k}^{\perp}\right\rangle=\sqrt{1-d_{k}}\left|r_{k}\right\rangle+$ $\sqrt{d_{k}}\left|r_{k}^{\perp}\right\rangle$.

Reference [5] shows that if the current state is in the $2 \times 2$ block containing the ground state of $H_{t}$, then the PEPS structure guarantees the probability of a successful projection onto $P_{t+1}$ is at least $\kappa\left(A^{t+1}\right)^{-2}$, where $\kappa\left(A^{t+1}\right)$ is the condition number of the matrix $A^{t+1}$. Assume for induction that we have already successfully prepared the (unique) ground state of $H_{t}$. We first attempt to project from this state onto the unique ground state of $H_{t+1}$ by measuring $\left\{P_{t+1}, P_{t+1}^{\perp}\right\}$. If this fails, we attempt to project back to the state we started from by measuring $\left\{P_{t}, P_{t}^{\perp}\right\}$ (a technique introduced by Marriott and Watrous [16] in the context of Quantum Merlin-Arthur (QMA) amplification). If this "rewind" measurement succeeds, then we are back where we started and can try again. What if the "rewind" measurement fails? By Lemma 1, we must be in the excited state from the same $2 \times 2$ block, so we can still try to project "forwards" with the same lower bound on the success probability, thus, iterating forwards and backwards measurements until success generates a Markov chain with successful projection onto the ground state of $H_{t+1}$ as the unique absorbing state. Moreover, the success probability in each step is bounded away from zero, so this process converges in polynomial time to the ground state of $H_{t+1}$.

\section{B. Preparing $G$-injective PEPS}

Consider the algorithm of the preceding section from the perspective of $G$-injective PEPS. An injective PEPS can always be viewed as a $G$-injective PEPS for the representation $\mathbb{1}$ of the trivial group. The algorithm starts from the state

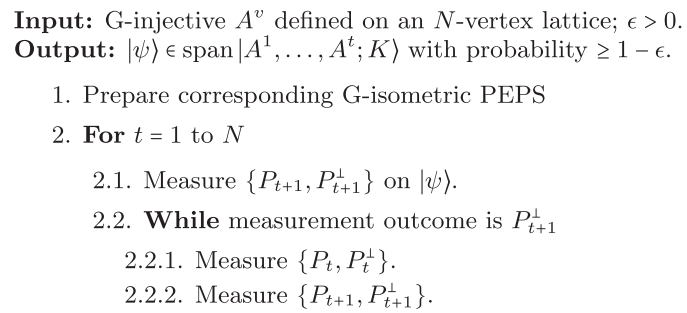

FIG. 2. Preparing a $G$-injective PEPS. $H_{t}(t=1 \ldots N)$ is the parent Hamiltonian for the $G$-injective PEPS $\left|A^{1} \ldots A^{t}\right\rangle, P_{t}$ the projector onto its ground-state subspace. Note that by specifying $A^{v}$, we are implicitly selecting a particular semiregular representation of $G$.

consisting of maximally entangled pairs between each site, and transforms this into the desired state by projecting onto the ground states of a sequence of injective parent Hamiltonians. But, the initial state is none other than the $G$-isometric PEPS corresponding to the representation $\mathbb{1}$ of the trivial group. This hints at a generalization of the algorithm to $G$-injective PEPS for arbitrary groups $G$ : start by preparing the corresponding $G$-isometric PEPS, and successively transform this into the desired $G$-injective PEPS by projecting onto the ground states of the sequence of $G$-injective parent Hamiltonians (see Fig. 2).

There are, however, two obstacles to implementing this approach. (i) The initial $G$-isometric PEPS can be a substantially more complicated many-body quantum state than the trivial product of maximally entangled pairs we must prepare in the injective case. (ii) Since $G$-injective parent Hamiltonians are topological, they have degenerate ground-state subspaces. But, the Marriott-Watrous "rewinding trick" [16] relies on the measurement projectors being one dimensional; it breaks down in general for higher-dimensional projectors.

There is a direct solution to (i). Reference [7] proves that, for any group $G$, the parent Hamiltonian of the $G$-isometric PEPS for the regular representation corresponds precisely to a quantum-double model $[9,10]$. But, Ref. [11] shows that ground states of quantum-double models can be generated exactly by a polynomial-size quantum circuit. We can therefore use this circuit to efficiently prepare the $G$-isometric PEPS for the regular representation of $G$. Even though the argument above only applies to regular representations of $G$, we have shown in Sec. I that a $G$-isometric PEPS for any semiregular representation is equivalent to the one for the regular representation (up to local isometries) by simply regrouping the tensors. Thus, the argument generalizes straightforwardly.

The second obstacle (ii) is more delicate. As described above, the Marriott-Watrous "rewinding" used in the injective case [5] works because the Hamiltonians $H_{t}$ and $H_{t+1}$ at each step have unique ground states, so that the back-and-forth measurement process is confined to a single $2 \times 2$ block. However, in the $G$-injective case, the Hamiltonians no longer have unique ground states, and there are multiple $2 \times 2$ blocks corresponding to different ground states. Thus, when we "rewind" a failed measurement, the backwards measurement could project us back into any superposition of states from any of the blocks corresponding to the ground-state subspace. Now, $A^{t+1}$ is only invertible on the $G$-symmetric subspace, so 
it necessarily has some zero eigenvalues. Hence, $\kappa\left(A^{t+1}\right)=\infty$ and the lower bound $\kappa\left(A^{t+1}\right)^{-2}=0$ on the probability of a successful forward measurement is useless. Although there may still exist some ground state $\left|\psi_{t}^{1}\right\rangle$ of $H_{t}$ which has positive probability of successful forward transition to a ground state of $H_{t+1}$, this does not rule out the existence of another ground state $\left|\psi_{t}^{2}\right\rangle$ of $H_{t}$ for which the probability of a successful forward transition is 0 . In the worst case, if a forward measurement fails and we end up in a state $\left|\varphi_{t+1}^{\perp}\right\rangle$, the rewinding step could have probability 1 of transitioning back to $\left|\psi_{t}^{2}\right\rangle$, so that we remain stuck forever bouncing back and forth between $\left|\psi_{t}^{2}\right\rangle$ and $\left|\varphi_{t+1}^{\perp}\right\rangle$.

To overcome this, we must show that if we start from the $G$-isometric state, then the structure of $G$-injective PEPS ensures that this situation can never occur. To prove this, we need the following technical lemma, which generalizes Lemma 2 of [5].

Lemma 2. Let $P_{t}$ and $P_{t+1}$ denote two projectors on the ground-state subspace of the partial PEPS parent Hamiltonains $H_{t}$ and $H_{t+1}$ for $\left|A^{1} \ldots A^{t}\right\rangle$ and $\left|A^{1} \ldots A^{t}, A^{t+1}\right\rangle$. The overlap $d_{k}$ between $P_{t}$ and $P_{t+1}$ (cf. Lemma 1) is lower bounded by $d_{\min } \geqslant \kappa\left(\left.A^{t+1}\right|_{S_{G}}\right)^{-2}$, where $\kappa\left(\left.A^{t+1}\right|_{S_{G}}\right):=$ $\sigma_{\max }\left(\left.A_{t+1}\right|_{S_{G}}\right) / \sigma_{\min }\left(\left.A_{t+1}\right|_{S_{G}}\right)$ is the condition number restricted to the $G$-symmetric subspace $S_{G}$.

Proof. The minimum overlap $d_{\text {min }}$ between projectors $P_{t}$ and $P_{t+1}$ is given by

$$
d_{\min }=\left.\min _{\left|\psi_{t}\right\rangle} \max _{\left|\psi_{t+1}\right\rangle}\left\langle\psi_{t} \mid \psi_{t+1}\right\rangle\right|^{2}
$$

where $\left|\psi_{t}\right\rangle$ and $\left|\psi_{t+1}\right\rangle$ are states in the respective ground-state subspaces $\operatorname{ker} H_{t}$ and $\operatorname{ker} H_{t+1}$. Now, ker $H_{t}$ is spanned by the partially constructed PEPS $\left|A^{1}, \ldots, A^{t} ; K\right\rangle$, with different boundary conditions $K$ giving different ground states. Thus, we can decompose any $\left|\psi_{t}\right\rangle \in \operatorname{ker} H_{t}$ as a linear combination of partial PEPS: $\left|\psi_{t}\right\rangle=\sum c_{k}\left|A^{1}, \ldots, A^{t} ; K^{k}\right\rangle . H_{t+1}$ is obtained from $H_{t}$ by replacing all the $G$-isometric local Hamiltonian terms at one vertex with the $G$-injective terms. So, applying $A_{t+1}$ to any $\left|A^{1}, \ldots, A^{t} ; K\right\rangle$ takes us to the next ground-state subspace. Therefore, the state

$$
\left|\varphi_{t+1}\right\rangle=\frac{A_{t+1}}{\left|\psi_{t}\right\rangle} \sqrt{\left\langle\psi_{t}\left|A_{t+1}^{\dagger} A_{t+1}\right| \psi_{t}\right\rangle}
$$

is contained in ker $H_{t+1}$. Choosing $\left|\psi_{t+1}\right\rangle=\left|\varphi_{t+1}\right\rangle$ in Eq. (5), we obtain the lower bound

$$
d_{\min } \geqslant \min _{\left|\psi_{t}\right\rangle}\left|\left\langle\psi_{t} \mid \varphi_{t+1}\right\rangle\right|^{2} \geqslant \min _{\left|\psi_{t}\right\rangle} \frac{\left|\left\langle\psi_{t}\left|A_{t+1}\right| \psi_{t}\right\rangle\right|^{2}}{\left\langle\psi_{t}\left|A_{t+1}^{\dagger} A_{t+1}\right| \psi_{t}\right\rangle} .
$$

It is immediate from the definition of $G$-injective PEPS that the ground states of $H_{t}$ are symmetric, so that the projector $P_{t}$ is supported on the symmetric subspace $S_{G}$. Thus, the minimization is over symmetric states and, recalling that w.l.o.g. $A_{t}$ is positive semidefinite, we obtain the claimed bound

$$
d_{\min } \geqslant \min _{\left|\psi_{t}\right\rangle} \frac{\left\langle\psi_{t}\left|A_{t+1}\right| s_{G} \mid \psi_{t}\right\rangle^{2}}{\left\langle\psi_{t}\left|A_{t+1}^{2}\right| S_{G}\right.}\left|\psi_{t}\right\rangle \geqslant \frac{\sigma_{\min }\left(A_{t+1} \mid S_{G}\right)^{2}}{\sigma_{\max }\left(\left.A_{t+1}\right|_{S_{G}}\right)^{2}}
$$

by the variational characterization of eigenvalues.

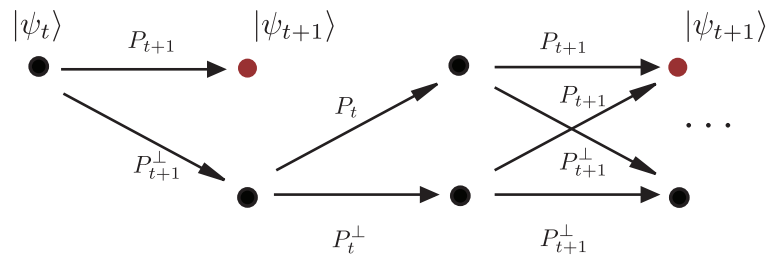

FIG. 3. (Color online) The sequence of outcomes of the binary measurements $\left\{P_{t}, P_{t}^{\perp}\right\}$ and $\left\{P_{t+1}, P_{t+1}^{\perp}\right\}$. We start in an eigenstate $\left|\psi_{t}\right\rangle$ of $P_{t}$, and want to transition to a state in the subspace $P_{t+1}$. With nonzero probability, the first measurement succeeds with outcome $P_{t+1}$. If it fails, we have prepared a state in the $P_{t+1}^{\perp}$ subspace. We "unwind" the measurement by measuring $\left\{P_{t}, P_{t}^{\perp}\right\}$ again. Upon repeating the $\left\{P_{t+1}, P_{t+1}^{\perp}\right\}$ measurement, we again have a nonzero probability of successfully obtaining the $P_{t+1}$ outcome. This is repeated until success.

\section{ANALYSIS}

Runtime. We are now in a position to establish the runtime of the algorithm given in Fig. 2. We start by bounding the failure probability of growing the partial PEPS by a single site. The proof of the following lemma is closely analogous to Lemma 3 in [5] and reproduced below.

Lemma 3. The measurement sequence depicted in Fig. 3 with the two projective measurements $\left\{P_{t}, P_{t}^{\perp}\right\}$ and $\left\{P_{t+1}, P_{t+1}^{\perp}\right\}$ has a failure probability bounded by

$$
p_{\text {fail }}(m)<\frac{1}{2 d_{\min } m}
$$

after $m$-subsequent measurement steps, where $d_{\min }=\min _{k} d_{k}$ is the minimal overlap between the eigenstates of $P_{t}$ and $P_{t+1}$.

Proof. Let $Q_{1}=P_{t+1}, Q_{0}=P_{t+1}^{\perp}$ and $R_{1}=P_{t}, R_{0}=$ $P_{t}^{\perp}$, in accordance with the notation in Lemma 1 . Hence, $Q_{1}$ projects on to the new ground-state subspace, whereas $R_{1}$ is the projector on to the old groundstate subspace. If we start in some state $|\psi\rangle=R_{1}|\psi\rangle$, the probability of failure of the measurement sequence depicted in Fig. 3 after $m$ steps is $p_{\text {fail }}(m)=\sum_{s_{1}, \ldots, s_{m}}$ $\operatorname{Tr}\left(Q_{0} R_{s_{m}} Q_{0} \ldots R_{s_{1}} Q_{0}|\psi\rangle\langle\psi| Q_{0} R_{s_{1}} \ldots Q_{0} R_{s_{m}} Q_{0}\right)$. Note that $\left[Q_{0} R_{s} Q_{0}, Q_{0} R_{p} Q_{0}\right]=0$ for all $s, p$. We can therefore rearrange this to express $p_{\text {fail }}(m)$ as the sum

$$
\begin{gathered}
\sum_{k=0}^{m}\left(\begin{array}{c}
m \\
k
\end{array}\right)\left\langle\psi\left|\left(Q_{0} R_{0} Q_{0}\right)^{2 k}\left(Q_{0} R_{1} Q_{0}\right)^{2(m-k)}\right| \psi\right\rangle \\
=\left\langle\psi\left|\left(\left(Q_{0} R_{0} Q_{0}\right)^{2}+\left(Q_{0} R_{1} Q_{0}\right)^{2}\right)^{m}\right| \psi\right\rangle .
\end{gathered}
$$

If we work in the eigenbasis of $Q_{1}$, the individual $2 \times 2$ block matrices take the form

$$
Q_{1}^{k}=\left(\begin{array}{ll}
1 & 0 \\
0 & 0
\end{array}\right), \quad R_{1}^{k}=\left(\begin{array}{cc}
d_{k} & \sqrt{d_{k}\left(1-d_{k}\right)} \\
\sqrt{d_{k}\left(1-d_{k}\right)} & 1-d_{k}
\end{array}\right) .
$$

Since $|\psi\rangle$ is left invariant by $R_{1}$, we have that $|\psi\rangle=$ $\sum_{k} c_{k}\left|r_{k}\right\rangle$, where in this basis every $\left|r_{k}\right\rangle=\left(\begin{array}{ll}\sqrt{d_{k}} & \left.\sqrt{1-d_{k}}\right)^{T}\end{array}\right.$ by Lemma 1 . We are therefore left with

$$
p_{\text {fail }}(m)=\sum_{k}\left|c_{k}\right|^{2}\left(1-d_{k}\right)\left[1-2 d_{k}\left(1-d_{k}\right)\right]^{m},
$$

with $d_{k} \in[0,1]$ and $\sum_{k}\left|c_{k}\right|^{2}=1$. 
Since $(1-x) \leqslant e^{-x}$, we may bound $\left(1-d_{k}\right)\left[1-2 d_{k}(1-\right.$ $\left.\left.d_{k}\right)\right]^{m} \leqslant\left(1-d_{k}\right) e^{-2 m d_{k}\left(1-d_{k}\right)}$. Furthermore, we have that $\left(1-d_{k}\right) e^{-2 m d_{k}\left(1-d_{k}\right)} \leqslant 1 / 2 m d_{k}$ by Taylor expansion. If we now choose the largest factor $\left(2 m d_{k}\right)^{-1} \leqslant\left(2 m d_{\text {min }}\right)^{-1}$, we can bound the total failure probability by Eq. (9).

We can use this to bound the overall runtime.

Theorem 4 (Runtime). Let $A^{v}$ be $G$-symmetric tensors defining a PEPS on an $N$-vertex lattice. A state in the subspace spanned by the corresponding $G$-injective PEPS $\left|A^{1} \ldots A^{N} ; K\right\rangle$ can be prepared on a quantum computer with probability $1-\epsilon$ in time $O\left(N^{4} \kappa_{G}^{2} \Delta^{-1} \epsilon^{-1}\right)$, with additional classical processing $O\left(N d^{6}\right) . \Delta=\min _{t}\left(\Delta_{t}\right)$ is the minimal spectral gap of the family of parent Hamiltonians $H_{t}$ for $\left|A^{1} \ldots A^{t}\right\rangle(t=1 \ldots N)$, and $\kappa_{G}=\max _{t} \kappa\left(\left.A^{t}\right|_{S_{G}}\right)$.

Proof. The algorithm in Fig. 2 first prepares the initial $G$-isometric PEPS, which can be done exactly in time $O(N \ln N)$ [11], and then transforms this step by step into the $G$-injective PEPS, with one step for each of the $N$ vertices of $\mathcal{G}$. Each step has a probability of failure $p_{\text {fail }}(m)$ if we repeat the back-and-forth measurement scheme $m$ times. We need to ensure that the total success probability is lower bounded by $\left[1-p_{\text {fail }}(m)\right]^{N} \geqslant 1-\epsilon$. Since $(1-x)^{N} \geqslant 1-N x$, we can use Lemma 3 to bound $\left[1-p_{\text {fail }}(m)\right]^{N} \geqslant 1-\frac{N}{2 m d_{\min }}$, so we want $N / 2 m d_{\min } \leqslant \epsilon$. Since $d_{\min } \geqslant \kappa_{G}^{-2}$ by Lemma 2 , we choose $m \geqslant N \kappa_{G}^{2} / 2 \epsilon$ at each step. We therefore need to perform $O\left(N^{2} \kappa_{G}^{2} \epsilon^{-1}\right)$ quantum phase estimation procedures, each of which has runtime $\tilde{O}\left(N^{2} / \Delta^{-1}\right)$ to ensure that we are able to resolve the energy gap of the parent Hamiltonian [17]. [Note that the notation $\tilde{O}(\ldots)$ suppresses more slowly growing terms such as $\exp (\sqrt{\ln N / \Delta})$.) The classical bookkeeping required to keep track of the Hamiltonians is the same as in [5]. Putting all this together, we arrive at the total runtime stated in the theorem.

\section{DISCUSSION}

We have shown how the Marriott-Watrous rewinding technique combined with the unique structure of $G$-injective PEPS can be used to transition from one state to the next (see Fig. 2), successively building up the desired quantum state, even when the state has topological order and the ground states are degenerate. A number of alternative techniques could potentially be substituted for the measurement rewinding approach used here. In each case, the key to proving an efficient runtime is our Lemma 2. In many cases, the existing results in the literature assume nondegenerate ground states, so would need to be generalized before they would apply to the topologically degenerate ground states considered here.

Standard adiabatic state preparation requires a polynomial energy gap along a continuous path joining the initial Hamiltonian with the final one. But, the "jagged adiabatic lemma" of Ref. [18] shows that such a path connecting a discrete set of gapped Hamiltonians always exists if the ground states are unique, and each has sufficient overlap with the next. This is precisely what we prove in Lemma 2. For the "injective" case of [5], this is sufficient to show that adiabatic state preparation is an efficient alternative to the "rewinding" technique. Our results suggest it may be possible to generalize the jagged adiabatic lemma to degenerate ground states.

More general are the methods of [19], which subsume the jagged adiabatic lemma and the Marriott-Watrous technique. The results in [19] do not immediately apply to degenerate ground states, but if they can be generalized they could potentially improve the polynomial dependence on the required error probability to a logarithmic one. Similarly, the quantum rejection sampling technique of [20] gives a quadratic improvement over Marriott-Watrous rewinding by a clever use of amplitude amplification. Finally, the spectral gap amplification technique of [21], which cites injective PEPS preparation [5] as a potential application, may also be applicable. In all three cases, the techniques would first need to be generalized to handle degenerate ground states. Our Lemma 2 would then imply efficiency of the resulting algorithm.

The conditions required for efficient preparation in Theorem 4 (inverse-polynomial scaling of the spectral gaps of the partial parent Hamiltonians, and polynomial scaling of the condition numbers of the PEPS projectors) are reminiscent of the conditions (local gap and local topological quantum order) required for stability of the spectral gap of local Hamiltonians [22]. It is also conjectured that the spectral gap of the parent Hamiltonian should be closely related to the condition number of the PEPS projectors. It would be interesting to understand better the relationships between these various conditions.

The technique introduced in this article, of constructing a complex many-body quantum state by starting from an easily constructable state and successively transforming it into the desired state, is very general. Although we have applied it here to $G$-injective PEPS, as a class of states including many important topological quantum states, our algorithm can be generalized to other classes of tensor network states, such as string-net models [23] and models constructed from Hopf algebras [24].

\section{ACKNOWLEDGMENTS}

D.P.G. and T.S.C. thank the Centro de Ciencias Pedro Pascual and the Petronilla facility, Benasque, where part of this work was done. They were funded by Spanish grants QUITEMAD, I-MATH, and Grant No. MTM2008-01366. T.S.C. was supported by a Juan de la Cierva fellowship, M.S. by Austrian SFB Grant No. FoQuS F4014, K.T. by an Erwin Schrödinger fellowship and Austrian FWF: J 3219-N16, F.V. by EU grants QUERG, and Austrian FWF SFB grants FoQuS and ViCoM.
[1] I. Bloch, J. Dalibard, and S. Nascimbène, Nat. Phys. 8, 267 (2012).

[2] R. Blatt and C. F. Roos, Nat. Phys. 8, 277 (2012).

[3] A. Aspuru-Guzik and P. Walther, Nat. Phys. 8, 285 (2012).
[4] A. A. Houck, H. E. Türeci, and J. Koch, Nat. Phys. 8, 292 (2012).

[5] M. Schwarz, K. Temme, and F. Verstraete, Phys. Rev. Lett. 108, 110502 (2012).

[6] F. Verstraete and J. I. Cirac, Phys. Rev. A 70, 060302(R) (2004). 
[7] N. Schuch, I. Cirac, and D. Peréz-García, Ann. Phys. (NY) 325, 2153 (2010).

[8] D. Poilblanc, N. Schuch, D. Peréz-García, and J. I. Cirac, Phys. Rev. B 86, 014404 (2012).

[9] A. Kitaev, Ann. Phys. (NY) 303, 2 (2003).

[10] N. Schuch, D. Perez-Garcia, and I. Cirac, Phys. Rev. B 84, 165139 (2011).

[11] M. Aguado and G. Vidal, Phys. Rev. Lett. 100, 070404 (2008).

[12] M. Nielsen and I. Chuang, Quantum Computation and Quantum Information (Cambridge University Press, Cambridge, 2000).

[13] E. Knill, G. Ortiz, and R. D. Somma, Phys. Rev. A 75, 012328 (2007).

[14] D. Berry, G. Ahokas, R. Cleve, and B. Sanders, Commun. Math. Phys. 270, 359 (2007).
[15] C. Jordan, Bull. Soc. Math. France 3, 103 (1875).

[16] C. Marriott and J. Watrous, Comput. Complex. 14, 122 (2005)

[17] A.W. Harrow, A. Hassidim, and S. Lloyd, Phys. Rev. Lett. 103, 150502 (2009).

[18] D. Aharonov and A. Ta-Shma, SIAM J. Comput. 37, 47 (2007).

[19] S. Boixo, E. Knill, and R. Somma, arXiv:1005.3034.

[20] M. Ozols, M. Roetteler, and J. Roland, in Proceedings of the 3rd Innovations in Theoretical Computer Science Conference, ITCS' 12 (ACM, New York, NY, 2012), pp. 290-308.

[21] R. Somma and S. Boixo, SIAM J. Comput. 42, 593 (2013).

[22] S. Michalakis, arXiv:1206.6900.

[23] N. Schuch (private communication).

[24] O. Buerschaper, M. Mombelli, M. Christandl, and M. Aguado, J. Math. Phys. 54, 012201 (2013). 